\title{
ERRATUM “THE SECOND CATALOG OF ACTIVE GALACTIC NUCLEI DETECTED BY THE FERMI LARGE AREA TELESCOPE” (2011, ApJ, 743, 171)
}

M. AckermanN ${ }^{1}$, M. Ajello ${ }^{1}$, A. Allafort ${ }^{1}$, E. Antolini ${ }^{2,3}$, W. B. Atwood ${ }^{4}$, M. Axelsson ${ }^{5,6,7}$, L. Baldini ${ }^{8}$, J. Ballet $^{9}$, G. Barbiellini ${ }^{10,11}$, D. Bastieri ${ }^{12,13}$, K. Bechtol ${ }^{1}$, R. Bellazzini ${ }^{8}$, B. Berenis ${ }^{1}$, R. D. Blandford ${ }^{1}$, E. D. Bloom ${ }^{1}$, E. Bonamente ${ }^{2,3}$, A. W. Borgland ${ }^{1}$, E. Bottacini ${ }^{1}$, A. Bouvier ${ }^{4}$, J. Bregeon $^{8}$, M. Brigida ${ }^{14,15}$, P. Bruel ${ }^{16}$, R. Buehler ${ }^{1}$, T. H. Burnett ${ }^{17}$, S. Buson ${ }^{12,13}$, G. A. Caliandro ${ }^{18}$, R. A. Cameron ${ }^{1}$, P. A. Caravero ${ }^{19}$, J. M. Casandjian ${ }^{9}$, E. Cavazzuti $^{20}$,

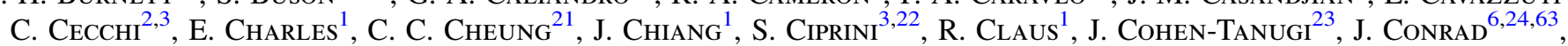
L. Costamante ${ }^{1}$, S. Cutini ${ }^{20}$, A. De Angelis ${ }^{25}$, F. de Palma ${ }^{14,15}$, C. D. Dermer ${ }^{26}$, S. W. Digel ${ }^{1}$, E. do Couto e Silva ${ }^{1}$, P. S. Drell ${ }^{1}$, R. Dubois ${ }^{1}$, L. Escande ${ }^{27}$, C. Favuzzi ${ }^{14,15}$, S. J. Fegan ${ }^{16}$, E. C. Ferrara ${ }^{28}$, J. Finke ${ }^{26}$, W. B. Focke ${ }^{1}$, P. Fortin ${ }^{16}$, M. Frailis ${ }^{25,29}$, Y. Fukazawa ${ }^{30}$, S. Funk ${ }^{1}$, P. Fusco ${ }^{14,15}$, F. Gargano ${ }^{15}$, D. Gasparrini ${ }^{20}$, N. Gehrels ${ }^{28}$, S. Germani ${ }^{2,3}$, B. Giebels ${ }^{16}$, N. Giglietto ${ }^{14,15}$, P. Giommi ${ }^{20}$, F. Giordano ${ }^{14,15}$, M. Giroletti ${ }^{31}$, T. Glanzman ${ }^{1}$, G. Godfrey ${ }^{1}$, I. A. Grenier ${ }^{9}$, J. E. Grove ${ }^{26}$, S. Guiriec ${ }^{32}$, M. Gustafsson ${ }^{12}$, D. Hadasch ${ }^{18}$, M. HaYashida ${ }^{1,33}$, E. Hays ${ }^{28}$, S. E. Healey ${ }^{1}$, D. Horan ${ }^{16}$,

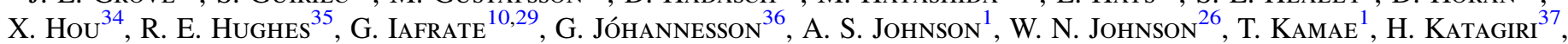

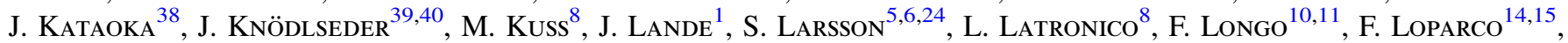

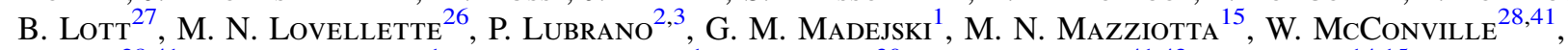

J. E. McEnery ${ }^{28,41}$, P. F. Michelson ${ }^{1}$, W. Mitthumsiri ${ }^{1}$, T. Mizuno ${ }^{30}$, A. A. Moiseev $^{41,42}{ }^{2}$ C. Monte ${ }^{14,15}$, M. E. Monzani ${ }^{1}$, E. Morettio ${ }^{6,7}$, A. Morseldi ${ }^{43}$, I. V. Moskalenko ${ }^{1}$, S. Murgia ${ }^{1}$, T. Nakamori ${ }^{38}$, M. Naumann-Godo ${ }^{9}$, P. L. Nolan ${ }^{1}$,

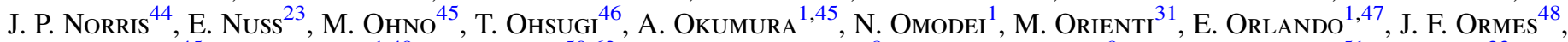
M. Ozaki ${ }^{45}$, D. Paneque ${ }^{1,49}$, D. Parent ${ }^{50,62}$, M. Pesce- Rollins ${ }^{8}$, M. Pierbattista ${ }^{9}$, S. Piranomonte ${ }^{51}$, F. Piron ${ }^{23}$, G. Pivato ${ }^{13}$, T. A. Porter ${ }^{1,1}$, S. Rainò ${ }^{14,15}$, R. Rando ${ }^{12,13}$, M. Razzano ${ }^{4,8}$, S. Razzaque ${ }^{5}$, A. Reimer ${ }^{1,52}$, O. Reimer ${ }^{1,52}$, S. Ritz ${ }^{4}$, L. S. Rochester ${ }^{1}$, R. W. Romani ${ }^{1}$, M. Roth ${ }^{17}$, D. A. Sanchez ${ }^{53}$, C. Sbarra ${ }^{12}$, J. D. Scargle ${ }^{54}$, T. L. Schalk ${ }^{4}$, C. SGRO ${ }^{8}$, M. S. Shaw ${ }^{1}$, E. J. Siskind ${ }^{55}$, G. Spandre ${ }^{8}$, P. Spinelli ${ }^{14,15}$, A. W. Strong ${ }^{47}$, D. J. Suson ${ }^{56}$, H. Tajima ${ }^{1,57}$, H. TAkahashi ${ }^{46}$, T. Takahashi ${ }^{4}$, T. Tanaka ${ }^{1}$, J. G. Thayer ${ }^{1}$, J. B. Thayer ${ }^{1}$, D. J. Thompson ${ }^{28}$, L. Tibaldo ${ }^{12,13}$, M. Tinivella $^{8}$, D. F. Torres ${ }^{18,58}$, G. Tosti ${ }^{2,3}$, E. Troja ${ }^{28,64}$, Y. UchiYama ${ }^{1}$, J. Vandenbroucke ${ }^{1}$, V. VAsileiou ${ }^{23}$, G. Vianello ${ }^{1,59}$,

V. Vitale ${ }^{43,60}$, A. P. WAite $^{1}$, E. Wallace ${ }^{17}$, P. WAnG ${ }^{1}$, B. L. Winer ${ }^{35}$, D. L. Wood ${ }^{61,62}$, K. S. Wood ${ }^{26}$, and S. Zimmer ${ }^{6,24}$

${ }^{\mathrm{i}}$ W. W. Hansen Experimental Physics Laboratory, Kavli Institute for Particle Astrophysics and Cosmology, Department of Physics and SLAC National Accelerator Laboratory, Stanford University, Stanford, CA 94305, USA

${ }_{3}^{2}$ Istituto Nazionale di Fisica Nucleare, Sezione di Perugia, I-06123 Perugia, Italy

${ }^{3}$ Dipartimento di Fisica, Università degli Studi di Perugia, I-06123 Perugia, Italy

${ }^{4}$ Santa Cruz Institute for Particle Physics, Department of Physics and Department of Astronomy and Astrophysics, University of California at Santa Cruz, Santa Cruz, CA 95064, USA

${ }^{5}$ Department of Astronomy, Stockholm University, SE-106 91 Stockholm, Sweden

${ }^{6}$ The Oskar Klein Centre for Cosmoparticle Physics, AlbaNova, SE-106 91 Stockholm, Sweden

${ }^{7}$ Department of Physics, Royal Institute of Technology (KTH), AlbaNova, SE-106 91 Stockholm, Sweden ${ }^{8}$ Istituto Nazionale di Fisica Nucleare, Sezione di Pisa, I-56127 Pisa, Italy

${ }^{9}$ Laboratoire AIM, CEA-IRFU/CNRS/Université Paris Diderot, Service d'Astrophysique, CEA Saclay, 91191 Gif sur Yvette, France

${ }^{10}$ Istituto Nazionale di Fisica Nucleare, Sezione di Trieste, I-34127 Trieste, Italy

${ }^{11}$ Dipartimento di Fisica, Università di Trieste, I-34127 Trieste, Italy

12 Istituto Nazionale di Fisica Nucleare, Sezione di Padova, I-35131 Padova, Italy

${ }^{13}$ Dipartimento di Fisica "G. Galilei", Università di Padova, I-35131 Padova, Italy

${ }^{14}$ Dipartimento di Fisica "M. Merlin" dell’Università e del Politecnico di Bari, I-70126 Bari, Italy

${ }^{15}$ Istituto Nazionale di Fisica Nucleare, Sezione di Bari, 70126 Bari, Italy

${ }^{16}$ Laboratoire Leprince-Ringuet, École polytechnique, CNRS/IN2P3, Palaiseau, France

${ }_{17}^{17}$ Department of Physics, University of Washington, Seattle, WA 98195-1560, USA

${ }^{18}$ Institut de Ciències de l'Espai (IEEE-CSIC), Campus UAB, 08193 Barcelona, Spain

${ }^{19}$ INAF-Istituto di Astrofisica Spaziale e Fisica Cosmica, I-20133 Milano, Italy

${ }^{20}$ Agenzia Spaziale Italiana (ASI) Science Data Center, I-00044 Frascati (Roma), Italy; elisabetta.cavazzuti@asdc.asi.it; sarac@slac.stanford.edu; gasparrini@asdc.asi.it

${ }^{21}$ National Research Council Research Associate, National Academy of Sciences, Washington, DC 20001, USA ${ }^{22}$ ASI Science Data Center, I-00044 Frascati (Roma), Italy

${ }^{23}$ Laboratoire Univers et Particules de Montpellier, Université Montpellier 2, CNRS/IN2P3, Montpellier, France

${ }^{24}$ Department of Physics, Stockholm University, AlbaNova, SE-106 91 Stockholm, Sweden

${ }^{25}$ Dipartimento di Fisica, Università di Udine and Istituto Nazionale di Fisica Nucleare, Sezione di Trieste, Gruppo Collegato di Udine, I-33100 Udine, Italy

${ }^{26}$ Space Science Division, Naval Research Laboratory, Washington, DC 20375-5352, USA; charles.dermer@nrl.navy.mil

${ }^{27}$ Université Bordeaux 1, CNRS/IN2p3, Centre d'Études Nucléaires de Bordeaux Gradignan, 33175 Gradignan, France; lott@cenbg.in2p3.fr ${ }^{28}$ NASA Goddard Space Flight Center, Greenbelt, MD 20771, USA

${ }^{29}$ Osservatorio Astronomico di Trieste, Istituto Nazionale di Astrofisica, I-34143 Trieste, Italy

${ }^{30}$ Department of Physical Sciences, Hiroshima University, Higashi-Hiroshima, Hiroshima 739-8526, Japan

${ }^{31}$ INAF Istituto di Radioastronomia, 40129 Bologna, Italy

${ }^{32}$ Center for Space Plasma and Aeronomic Research (CSPAR), University of Alabama in Huntsville, Huntsville, AL 35899, USA

${ }^{33}$ Department of Astronomy, Graduate School of Science, Kyoto University, Sakyo-ku, Kyoto 606-8502, Japan

${ }_{34}^{34}$ Centre d'Études Nucléaires de Bordeaux Gradignan, IN2P3/CNRS, Université Bordeaux 1, BP120, F-33175 Gradignan Cedex, France

${ }^{35}$ Department of Physics, Center for Cosmology and Astro-Particle Physics, The Ohio State University, Columbus, OH 43210, USA ${ }^{36}$ Science Institute, University of Iceland, IS-107 Reykjavik, Iceland

${ }^{37}$ College of Science, Ibaraki University, 2-1-1, Bunkyo, Mito 310-8512, Japan 


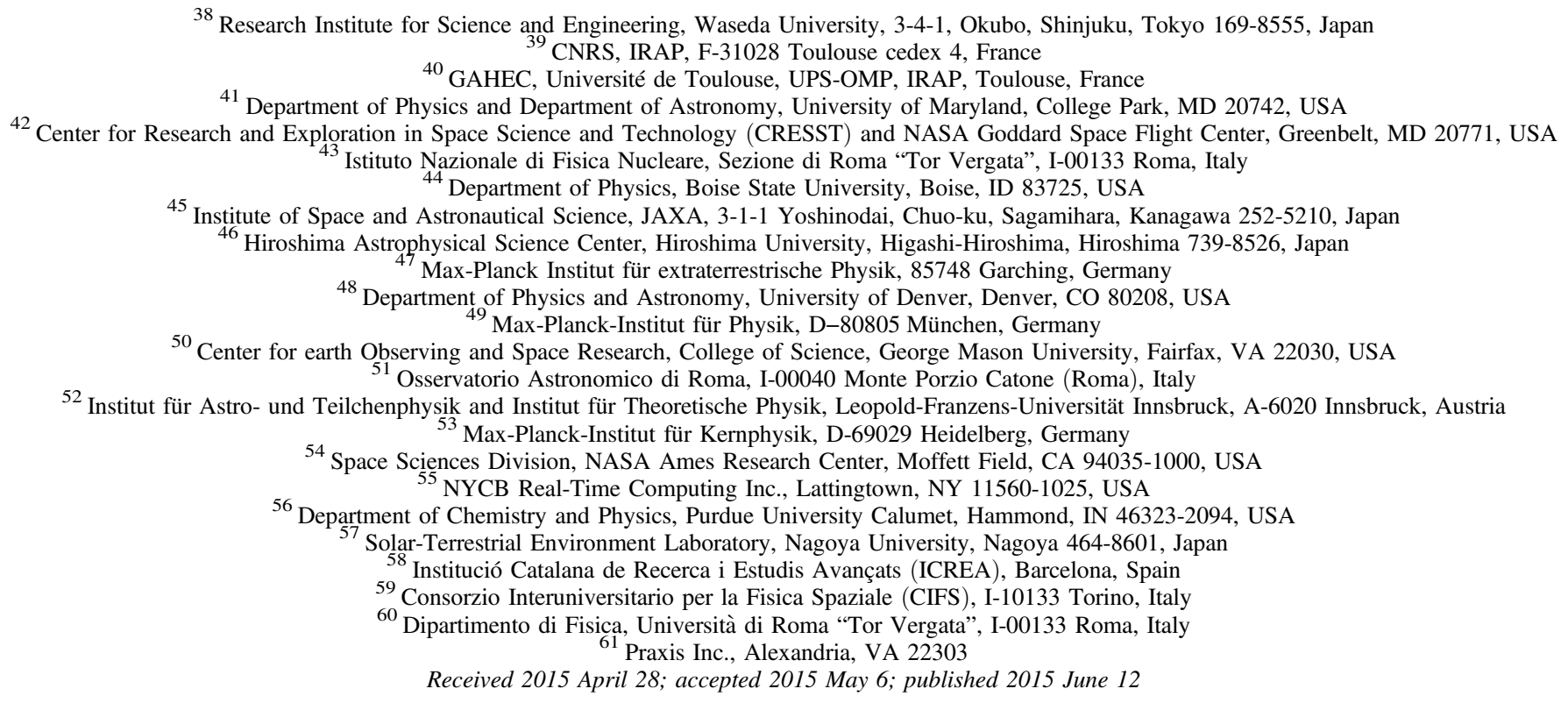

The code developed for the Likelihood-Ratio (LR) method used in the counterpart association procedure of the 2LAC catalog and described in Section 3.2 of the published paper contained an error in the epoch of the celestial coordinates used in the sky simulations. After the cross correlations, which were performed in the J2000.0 epoch, the coordinates of the fake sources were erroneously precessed to B1950.0. This affected the evaluation of the LR values of the fake skies and in turn the computation of the reliability function $R\left(L R_{i j}\right)$. The reliability curves were shifted to lower LR, resulting in higher association probabilities at a given LR. The corrected entries for Tables 1 (showing the LR critical value $L R_{c}$, i.e., the threshold where the reliability becomes greater than $80 \%$ for the different surveys) and 2 (comparing the results of the different association methods) are given below. The PMN survey has been removed since, with the corrected coordinate epoch, it does not bring any new association on its own.

In total, 88 former $2 \mathrm{LAC}$ sources have corrected association probabilities below 0.8 . These are widely distributed in Galactic latitude. A comparison between the initial and corrected census is given in Table 3. The revised 2LAC lists 929 sources with Galactic latitude $|b|>10^{\circ}$, with 10 double associations. The revised 2LAC Clean Sample now includes 827 sources (instead of 886) divided into 303 FSRQs, 381 BL Lacs, 124 blazars of unknown type, and 19 non-blazar AGNs. A total of 544 sources are now associated by all three association methods used in the 2LAC work, 507 being in the Clean 2LAC Sample.

Three non-blazar sources, formerly associated in the 2LAC catalog, have dropped off the revised 2LAC list: Fornax A, PKS 0943 -73 , and ESO 323-77. We also note that 2FGL J0114.7+1326 was erroneously associated, due to a typographical error, with the BL Lac GB6 J0114+1325, while the correct association is with the FSRQ GB6 J0115+1324. That association is now reported in the revised version of $2 \mathrm{LAC}$.

Table 1

Initial and Corrected Values of $L R_{c}$ for the Catalogs Used for 2LAC Associations

\begin{tabular}{lcc}
\hline \hline Survey & initial $\log L R_{c}$ & corrected $\log L R_{c}$ \\
\hline NVSS & -0.28 & 2.08 \\
SUMSS & 0.79 & 1.92 \\
RASS & 1.71 & 3.03 \\
AT20G & 2.91 & 6.20 \\
\hline
\end{tabular}

Table 2

Comparison of Association Methods (Correction of Table 2 in 2LAC)

\begin{tabular}{lcccccccccc}
\hline \hline Sample & Total & $N_{\text {false }}$ & Bayesian & $N_{\text {false }}$ & $\mathrm{N}_{S}$ & LR & $N_{\text {false }}$ & $\mathrm{N}_{S}$ & $\log N-\log S$ & $N_{\text {false }}$ \\
\hline All & 929 & 18.5 & 846 & 12.5 & 84 & 643 & 75.5 & 26 & 763 & 22.7 \\
Clean Sample & 827 & 14.7 & 754 & 9.1 & 64 & 594 & 69.0 & 24 & 691 & 19.1 \\
\hline
\end{tabular}

\footnotetext{
62 Resident at Naval Research Laboratory, Washington, DC 20375, USA.

${ }^{63}$ Royal Swedish Academy of Science Research Fellow, funded by a grant from the K. A. Wallenberg Foundation.

${ }^{64}$ NASA Postdoctoral Program Fellow, USA.
} 
Table 3

Comparison of Association Results between Initial and Corrected 2LAC Catalogs

\begin{tabular}{lcccc}
\hline \hline Sample & $\begin{array}{c}\text { 2FGL sources } \\
\text { ass. with AGNs }\end{array}$ & AGNs in & 2FAC & AGNs at \\
& 1095 & 1121 & $10^{\circ}$ & Clean Sample \\
\hline Initial & 1019 & 1029 & 929 & 104 \\
Corrected & 76 & 92 & 886 & 100 \\
\hline Sources affected & & & 427 \\
\hline
\end{tabular}

